\title{
Mesoporous Carbon Single-Crystals from Organic-Organic
}

\section{Self-assembly}

Fuqiang Zhang, Dong Gu, Ting Yu, Fan Zhang, Songhai Xie, Lijuan Zhang, Yonghui Deng, Ying Wan, Bo Tu and Dongyuan Zhao*

Department of Chemistry, Shanghai Key Laboratory of Molecular Catalysis and Innovative Materials, Fudan University, Shanghai 200433, P. R. China

Email: dyzhao@fudan.edu.cn

\section{Synthesis details:}

Mesoporous carbon materials were synthesized by using triblock copolymer as a template and phenol/formaldehyde as a carbon precursor. Firstly, $2.0 \mathrm{~g}(21 \mathrm{mmol})$ of phenol and $7.0 \mathrm{ml}$ of formaldehyde solution $(40 \mathrm{wt} \%, 100 \mathrm{mmol})$ were dissolved in 50 $\mathrm{ml}(5 \mathrm{mmol})$ of $0.1 \mathrm{M} \mathrm{NaOH}$ solution. Then the mixture was stirred at $70^{\circ} \mathrm{C}$ for 30 min. A clear precursor solution (about $60 \mathrm{ml}$ ) was obtained, denoted as Solution-P. FDU-16: For a typical synthesis of mesoporous carbon FDU-16, 5.6 g of F127 ( 0.44 mmol) was dissolved in $50 \mathrm{ml}$ of water. Then $60 \mathrm{ml}$ of Solution-P was added into the above solution, and a clear solution was obtained. The solution turned dark red as stirred at $66^{\circ} \mathrm{C}$ for about $3 \mathrm{~h}$ with a rate of $\sim 300 \mathrm{rpm}$. Yellow precipitation was observed after about $48 \mathrm{~h}$. The mixture was continuously stirred at $66^{\circ} \mathrm{C}$ for additional $72 \mathrm{~h}$ and then stirred at $70^{\circ} \mathrm{C}$ for another $24 \mathrm{~h}$. The final product was collected by sedimentation separation and filtration, washed with water and dried in air. The obtained sample was calcined at $700^{\circ} \mathrm{C}$ for $3 \mathrm{~h}$ in nitrogen flow to obtain mesoporous carbon FDU-16. The heating rate was fixed at $1^{\circ} \mathrm{C} / \mathrm{min}$

FDU-15: For a typical synthesis of mesoporous carbon FDU-15, $2.1 \mathrm{~g}$ of P123 (0.36 $\mathrm{mmol})$ and $3.5 \mathrm{~g}$ of F127 (0.28 mmol) was dissolved in $50 \mathrm{ml}$ water. Then $60 \mathrm{ml}$ of Solution-P was added into the above solution. The solution turned dark red as stirred at $66^{\circ} \mathrm{C}$ for about $3 \mathrm{~h}$. The mixture was continuously stirred at $66^{\circ} \mathrm{C}$ for additional $120 \mathrm{~h}$ and then stirred at $70^{\circ} \mathrm{C}$ for $24 \mathrm{~h}$. The final products were collected and calcined as FDU-16, FDU-15 were then obtained. 

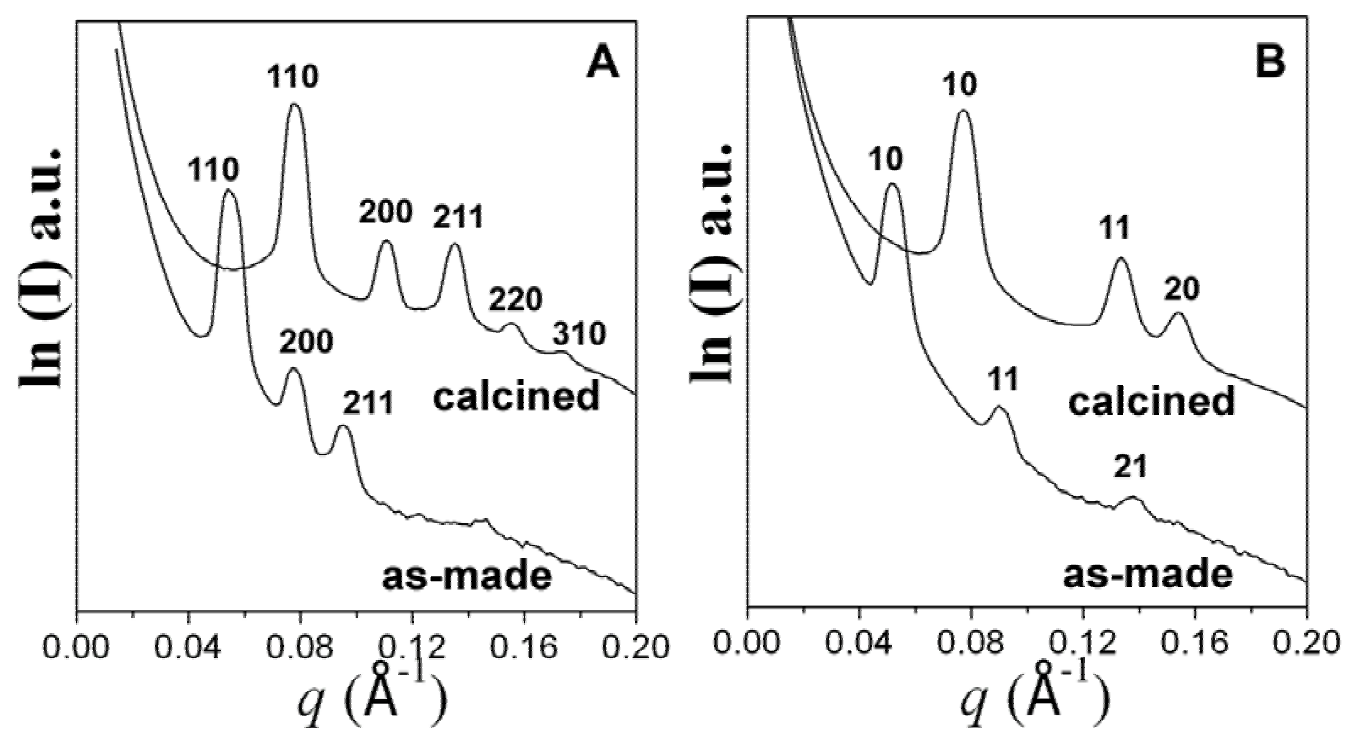

Figure 1. SAXS patterns of FDU-16 single crystals (A) and discus-like FDU-15 (B).

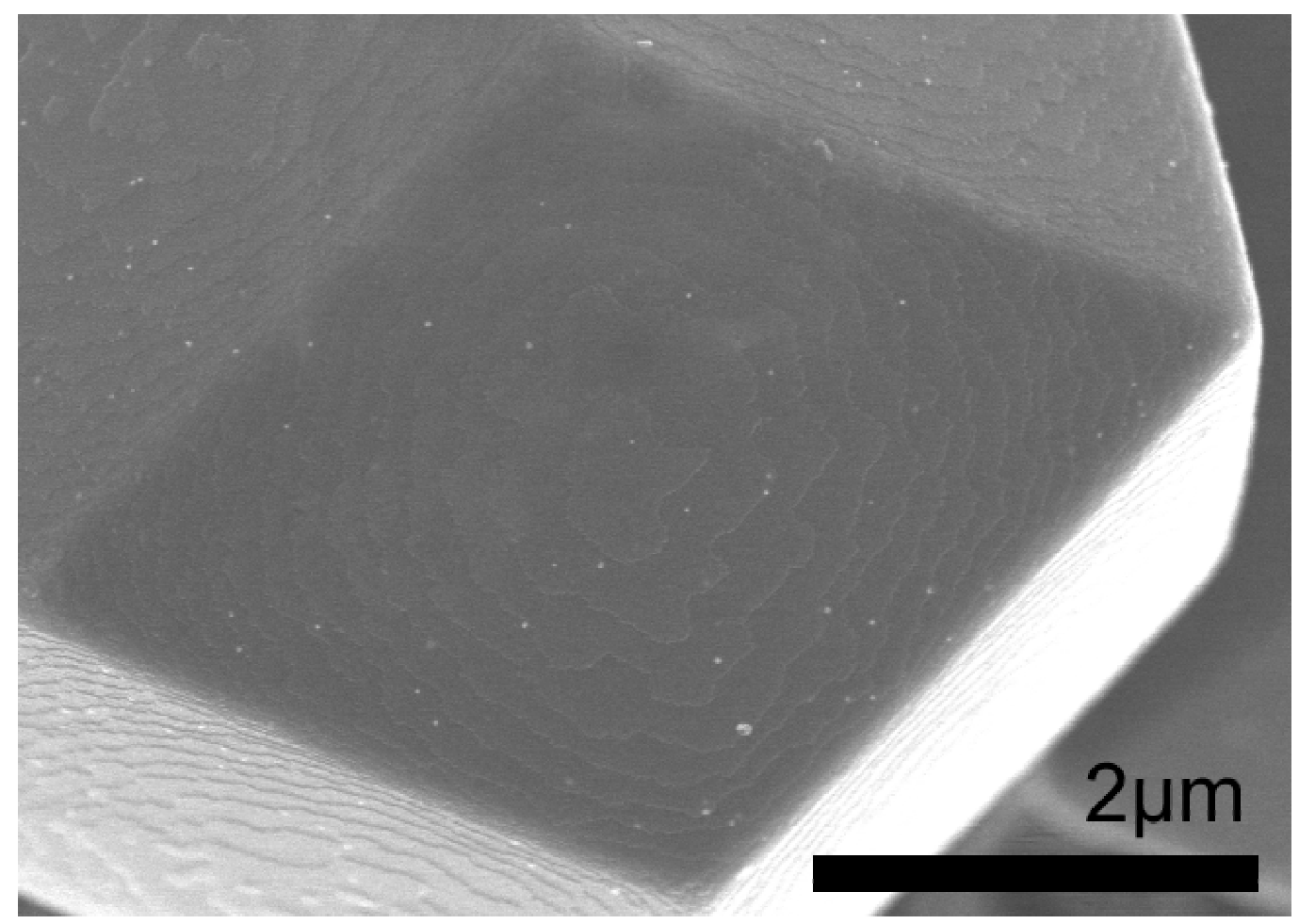

Figure 2. SEM image of mesoporous carbon FDU-16 after calcined at $700^{\circ} \mathrm{C}$ in Nitrogen. 


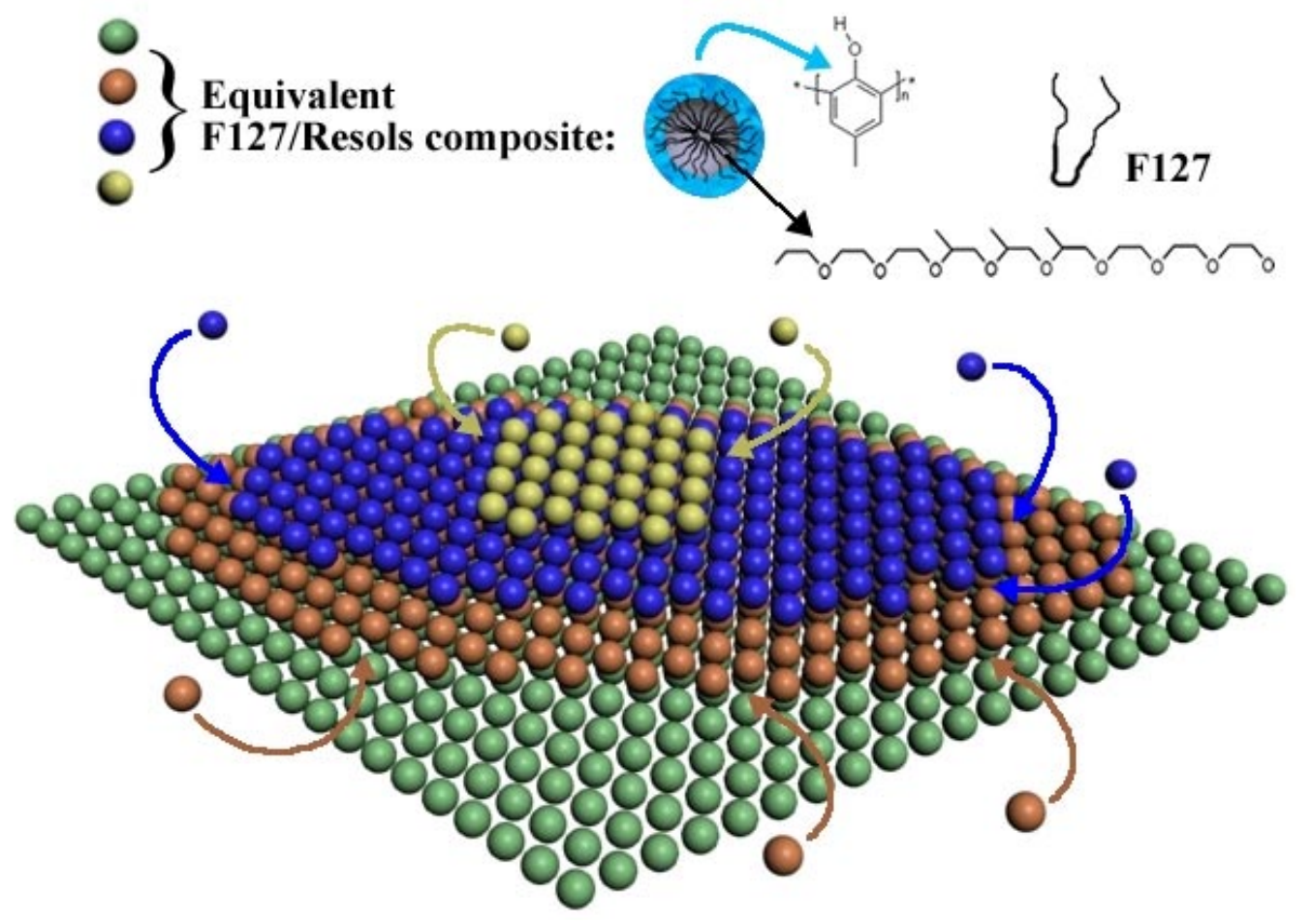

Figure 3. Scheme of layer-by-layer growth from $\{110\}$ planes of FDU-16. 


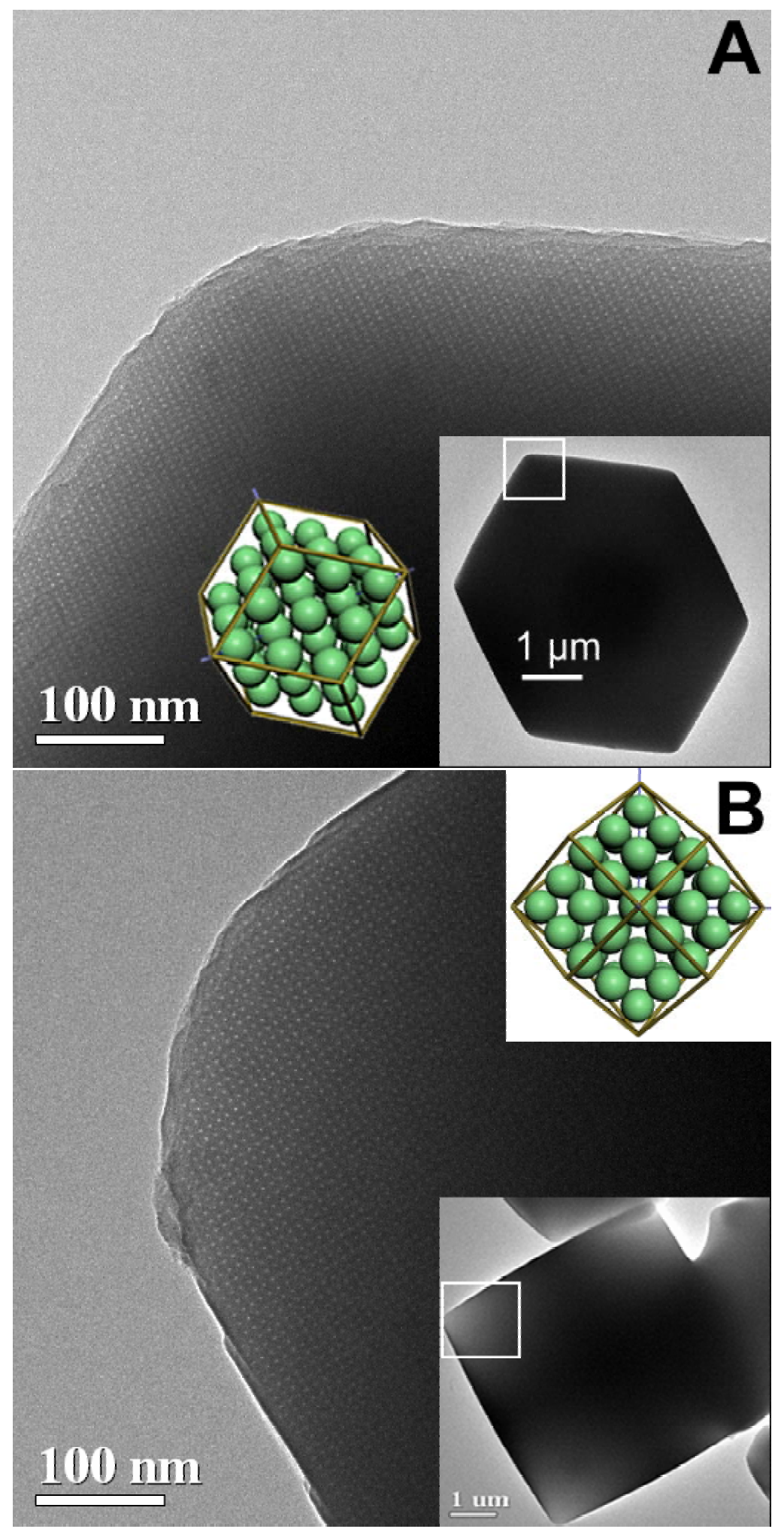

Figure 4. TEM image of FDU-16 single crystals viewed along [110] (A) and [100] (B) directions. Insets are the image of low magnification and structural models. The rectangle in each inset shows where the corresponding large image was taken from. 


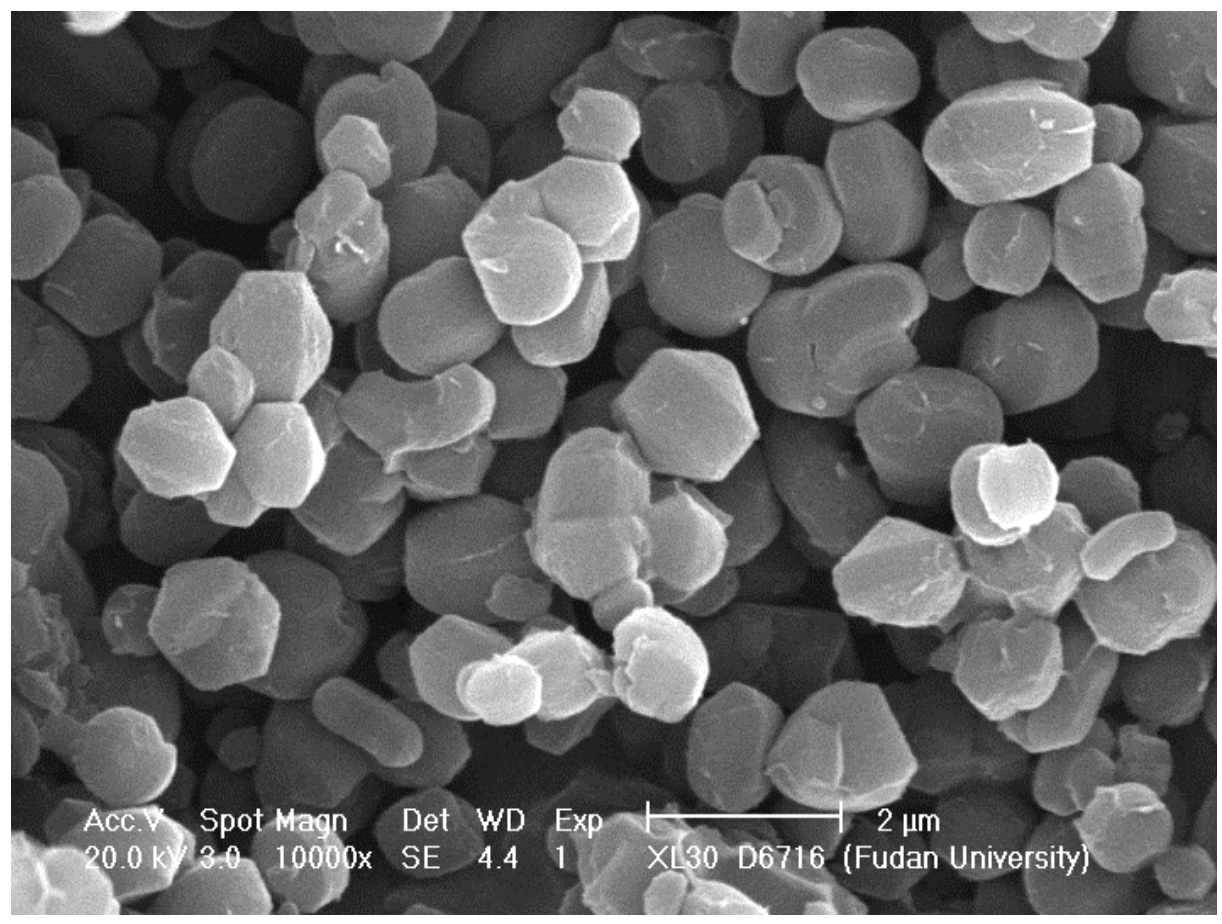

Figure 5. SEM image of mesoporous carbon FDU-15. 

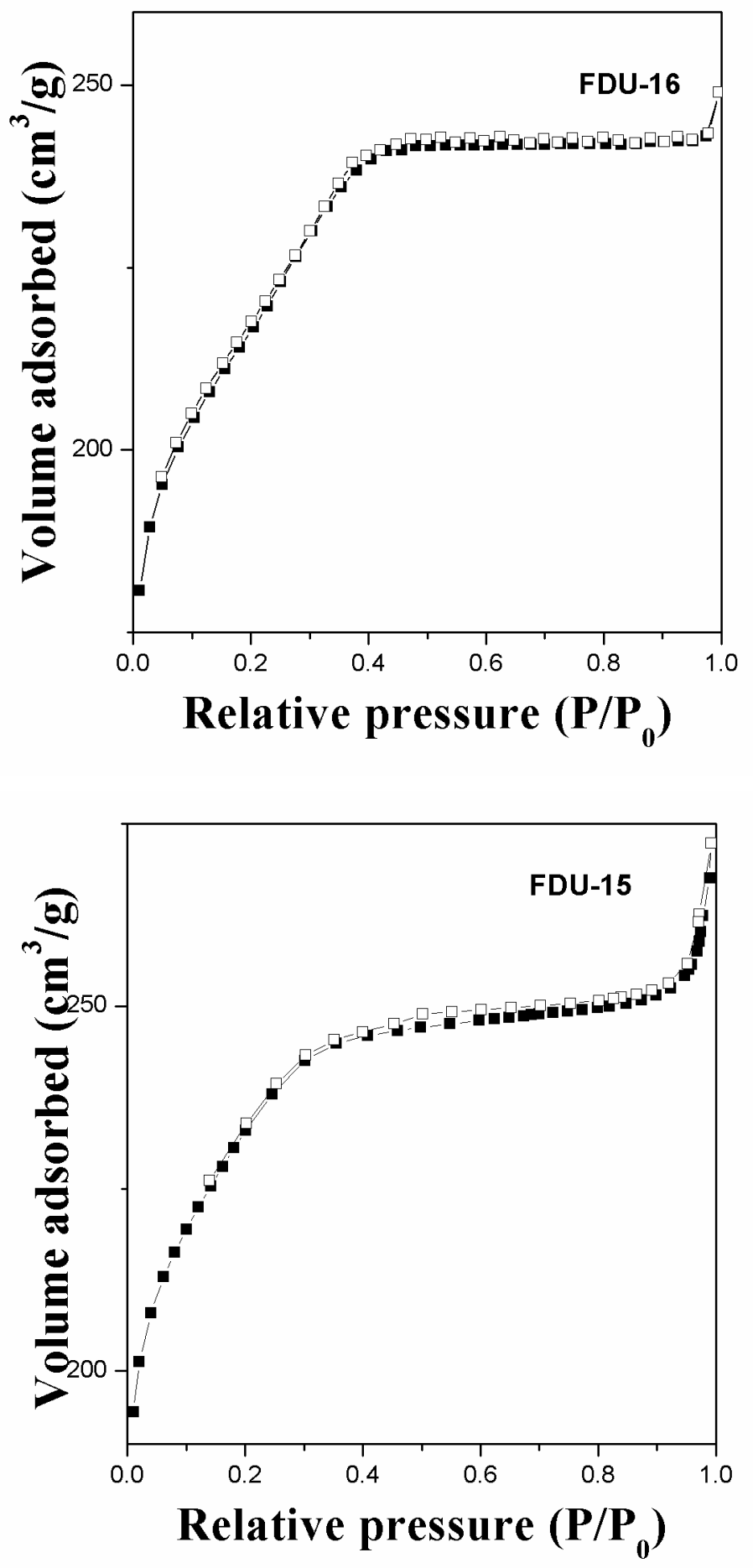

Figure 6. Nitrogen sorption isotherms of mesoporous carbon FDU-15 and FDU-16. 